Bio-grafia Escritos sobre la Biologia y su Enseñanza.

Edición Extra-Ordinaria. ISSN 2027-1034 P.p 705 - 714

Memorias del VII Encuentro Nacional de Experiencias en la Enseñanza de la Biología y la Educación Ambiental y II Congreso Nacional de Investigación en la Enseñanza de La Biología

\title{
LOS PROBLEMAS DE TRABAJO ABORDADOS EN LA ENSEÑANZA DE LA BIOLOGÍA, UNA MIRADA DESDE EL ESTADO DEL ARTE DE LA REVISTA THE AMERICAN BIOLOGYTEACHER 2007.
}

\section{WORK PROBLEMS APPROACHED IN THE TEACHING OF BIOLOGY. A LOOK FROM THE STATE-OF-ART OF THE AMERICAN BIOLOGY TEACHER MAGAZINE 2007}

\author{
Analida Altagracia Hernandez Pichardo ${ }^{1}$ \\ Mónica Alexandra Correa Sánchez ${ }^{2}$ \\ Yeimy Andrea Guerra Tellez ${ }^{3}$
}

\section{Resumen}

Se presentan los resultados de un estudio, cuyo objetivo consistió en caracterizar las publicaciones de la revista especializada en Enseñanza de la Biología The American Biology Teacher, para el año 2007. Esta investigación se orientó por un enfoque cualitativo, interpretativo, en la estrategia de investigación documental y modalidad de estado del arte; usando el Resumen Analítico Educativo (RAE) como herramienta de sistematización de la información, las categorías abordadas en su totalidad fueron: tipo de documento, sección de la revista, titulo, autor lugar de trabajo, publicación, palabras clave, problema objeto de la publicación, síntesis, fuente, problema, objetivo, población, aspectos metodológicos, conclusiones y tipo de trabajo. Para efectos de esta ponencia, se presentan los resultados de la categoría correspondiente a problema objeto de la publicación para esta categoría se eligieron siete sub-categorias que son: trabajos prácticos, enseñanza/aprendizaje de conceptos específicos, actitudes hacia las tematices biológicas, aspectos axiológicos en la Enseñanza de la Biología, naturaleza de las ciencias, evaluación, formación del profesorado. El estudio muestra que la tendencia mayoritaria es la presentación de trabajos prácticos lo que nos permite pensar que puede estarse constituyendo como un posible campo de investigación en la Enseñanza de la Biología.

\footnotetext{
${ }^{1}$ Universidad Pedagógica Nacional, Grupo de Investigación Conocimiento Profesional del Profesor de Ciencias (CPPC) anaherpi@hotmail.com

22 Universidad Pedagógica Nacional, Grupo de Investigación Conocimiento Profesional del Profesor de Ciencias (CPPC) macs1986@hotmail.com

${ }_{3}^{3}$ Universidad Pedagógica Nacional, Grupo de Investigación Conocimiento Profesional del Profesor de Ciencias (CPPC) yeimygrr@gmail.com
} 
Memorias del VII Encuentro Nacional de Experiencias en la Enseñanza de la Biología y la Educación Ambiental y II Congreso Nacional de Investigación en la Enseñanza de la Biología

\section{Abstract}

The results of a study were presented whose objective consisted in characterizing the publications of the specialized magazine of biology teaching The American Biology Teacher, for the year 2007. This research was oriented by a qualitative, interpretative approach in the strategy of documental research and the state-of-art modality using the Analytical Summary Educational (RAE) as a systematization tool of the information. The categories approached in its totality were: type of document, section of the magazine, title, author, workplace, publication, keywords, publication object issue, synthesis, source, problem, objective, population, methodological aspects, conclusions and work type. For the purposes of this brief, the results of the respective category are presented for the publication object issue. For this category seven subcategories were chosen: practical works, teaching/learning of specific concepts, attitude towards biological thematic, axiological aspects in the teaching of biology, science nature, evaluation and formation of faculty. The study shows that the greater tendency is the presentation of practical works which allows us to think that it may be constituting itself as a possible field of investigation in the teaching of biology.

Palabras Clave: Enseñanza de la Biología, Conocimiento Profesional del Profesor de Ciencias, Conocimiento Didáctico del Contenido, Campo de Conocimiento, Estado del arte, problemas abordados en la Enseñanza de la Biología.

Key Words: Teaching of biology, professional knowledge of the science teacher, didactic knowledge of the content, field of knowledge, state-of-art, issues approached in the teaching of biology.

\section{Introducción}

El Grupo de Investigación Conocimiento Profesional del Profesor de Ciencias (CPPC), adscrito al Departamento de Biología de la Universidad Pedagógica Nacional (Colombia), llevó a cabo un proyecto de investigación en dos fases: El estado del arte sobre el campo de conocimiento de la Enseñanza de la Biología (Valbuena et.al 2010) y Estado del arte de las características del campo del conocimiento sobre la enseñanza de la Biología Fase II (Hernández et.al 2011). Dichos proyectos partieron de la premisa de que las ciencias propiamente dichas son consideradas campos de conocimiento consolidados, ahora ¿puede considerarse la Enseñanza de la Biología un campo de conocimiento?. 
Bio-grafia Escritos sobre la Biologia y su Enseñanza.

Edición Extra-Ordinaria. ISSN 2027-1034 P.p 705 - 714

Memorias del VII Encuentro Nacional de Experiencias en la Enseñanza de la Biología y la Educación Ambiental y II Congreso Nacional de Investigación en la Enseñanza de la Biología

Para responder a esta pregunta el grupo (CPPC), realizó un Estado del arte que comprendió el estudio de revistas académicas en el período de 2004 a 2008 que abordan temáticas relacionadas con la Enseñanza de la Biología (EB). Los resultados de esta investigación, permitieron evidenciar la falta de elementos consolidados que indiquen que la EB es hoy un campo de conocimiento, no obstante, se visibilizaron elementos favorables a la constitución futura del mismo; esta investigación permitió evidenciar las características de lo que hoy se dice y se hace respecto a la Enseñanza de la Biología.

En concordancia con lo anterior, es posible observar la importancia de la sistematización de los trabajos relacionados con la Enseñanza de la Biología, como se ha venido adelantando en los trabajos de Valbuena et.al (2010), Hernández et.al (2011), Correa (2012) entre otros. Las investigaciones mencionadas encontraron que a nivel internacional existen dos revistas especializadas en la Enseñanza de la Biología: Journal of BiologicalEducation y The American BiologyTeacher, es esta última la base de la presente discusión en un aspecto puntual: los problemas de estudio abordados en las publicaciones que tratan la Enseñanza de la Biología.

Esta caracterización aporta elementos específicos al Conocimiento Didáctico del Contenido Biológico (CDCB) en tanto se evidencia la falta de elementos históricos acerca de la Enseñanza de la Biología, además se observa que esta se ha tratado sin ninguna especificidad siendo abordada desde la Enseñanza de las Ciencias o en la enseñanza específica de algunas de sus aéreas disciplinares, por ejemplo: la Botánica, la Genética, la Biotecnología, así, reconocer cuales son los problemas objeto de publicación define unas tendencias claras que pueden derivar en líneas de investigación particulares e independientes que apunten a la consolidación de lo que es hoy una emergente Didáctica de la Biología.

\section{Metodología}

La metodología que orientó esta investigación se enmarca en el enfoque cualitativo interpretativo, tomando como referencia la estrategia de análisis documental en la modalidad de estado del arte, en el cual se empleó el resumen analítico educativo RAE, como herramienta para la síntesis y procesamiento de los datos obtenidos de las diferentes publicaciones. La investigación se desarrolló en tres fases: contextualización, clasificación y categorización; para esta última fase se hace uso de la matriz de análisis propuesta por Valbuena, et.al (2010), que incluye las siguientes categorías: problema objeto de la publicación, composición del equipo de trabajo, sección de la revista, autores, aspectos biológico abordado, tipo de trabajo, población objeto de estudio, metodología y fuentes. 
Memorias del VII Encuentro Nacional de Experiencias en la Enseñanza de la Biología y la Educación Ambiental y II Congreso Nacional de Investigación en la Enseñanza de la Biología

Para la fase de contextualización se realiza la delimitación del problema, a partir de los hallazgos de Valbuena et.al (2010), en donde se muestra que de las revistas que publican estudios relacionados con Enseñanza de la Biología solo dos se especializan en esta temática; por lo tanto, se escoge la revista norteamericana The American BiologyTeacher para el año de 2007 y cuatro de sus secciones: Article (articulo), Inquiry\&l nvestigation (consulta e investigación), Quick Fix (Arreglo rápido), How do toit (Como hacerlo), dando como resultado un universo constituido por 47 artículos. Para la fase de clasificación se realiza un RAE por cada artículo procedente de la revista The American BiologyTeacher, 2007. Se hace uso del formato empleado por (Hernández et.al 2011) que presenta algunas modificaciones de la versión inicial usada en la primera fase del proyecto. La fase de elaboración de los Resúmenes Analíticos Educativos contó con una fase de triangulación en la que participaron integrantes del grupo de investigación (CPPC).

Para la fase de categorización se realizó la sistematización de la información aportada por los RAE, con la consecuente caracterización de cada una de las categorías y sub-categorías que emergieron en la matriz de sistematización diseñada Valbuena et al (2010).

\section{Resultados Y Discusión.}

\section{Acerca de la categoría problema objeto de la publicación}

Los problemas abordados en las publicaciones se relacionan con 14 subcategorías, dentro de las cuales se encuentra: trabajo practico, enseñanza/aprendizaje de conceptos específicos, Ideas previas/ Representaciones/Concepciones, factores personales y contextuales en la enseñanza de la Biología, Tecnologías de la información y la comunicación, libros de texto, lenguaje y aprendizaje, actitudes hacia las temáticas biológicas, aspectos axiológicos, naturaleza de las ciencias, evaluación, formación de profesores y no presenta.

En esta categoría se puede observar como la tendencia mayoritaria se ubica en las publicaciones relacionadas con las sub-categorías de trabajos prácticos (TP) y con la enseñanza/aprendizaje de conceptos específicos, convirtiéndose en las líneas prioritarias de las publicaciones relacionadas con la enseñanza de la Biología. Los trabajos prácticos: reportan un mayor número de ejercicios de laboratorio, mientras que las prácticas de campo y ejercicios de lápiz y papel se encuentra en un porcentaje notoriamente menor. Dentro de las prácticas de 
Bio-grafia Escritos sobre la Biologia y su Enseñanza.

Edición Extra-Ordinaria. ISSN 2027-1034 P.p 705 - 714

Memorias del VII Encuentro Nacional de Experiencias en la Enseñanza de la Biología y la Educación Ambiental y II Congreso Nacional de Investigación en la Enseñanza de la Biología

laboratorio se encuentra el de la autoría de Weaver, D (2007), quien describe el diseño de una práctica de laboratorio que muestra técnicas de cultivo de tejidos de células cardiacas en embriones de pollo.

Los estudios desarrollados en el marco de los trabajos prácticos constituyen un eje nuclear en la enseñanza de la Biología, lo que se corresponde con los trabajos abordados en la Didáctica de las ciencias. En comparación con el trabajo de Duran (2010) quien aborda la misma revista para el periodo de 2008, los trabajos prácticos son en ambas ocasiones la tendencia mayoritaria.

Es comprensible que los trabajos prácticos sean la tendencia mayoritaria respecto a la categoría de problema objeto de la publicación en tanto estos constituyen hoy en día una de las actividades más importantes que se realizan en la Enseñanza de las Ciencias ya que permiten perseguir gran variedad de objetivos y finalidades; lo cual posibilita una mejor comprensión de los conceptos científicos; plantear y contrastar hipótesis; el manejo de instrumentos y técnicas de laboratorio; la aplicación de estrategias para la resolución de problemas teóricos o prácticos (Caamaño, 2004).

El trabajo realizado por Correa (2012) presenta una perspectiva más amplia en términos temporales así, como en el número de publicaciones revisadas en el marco de los trabajos prácticos; en donde muestra como se han generado dos tendencias de investigación: una hacia la presentación de los protocolos y actividades, la otra hacia la problematización del uso de los trabajos prácticos.

Para el caso específico de este estudio solo se encontraron publicaciones acerca de la primera tendencia, es decir, que para el año de 2007 en la revista The America Biology Teacher, se está limitando a la descripción de experiencias, sin que hayan ejercicios de problematización de los mismos. La tendencia por el estudio de los trabajos prácticos hace pensar que se está conformando como una línea de investigación propia en la Enseñanza de la Biología.

En la sub-categoría Enseñanza/Aprendizaje de Conceptos específicos, se encuentra que los trabajos presentan protocolos o actividades para la enseñanza de conceptos que hacen parte de un eje temático en Biología, por ejemplo, Coleman, S; Jensen, J (2007), abordan el diseño de actividades experimentales para la enseñanza de la selección sexual, como pauta para la comprensión de la enseñanza de la evolución. Otra tendencia en cambio aborda el eje temático como tal, a partir de la incorporación de técnicas, estrategias o recursos novedosos para su enseñanza/aprendizaje como el caso del artículo de Bixler, A (2007) que discute y propone el uso de la ciencia ficción para la enseñanza de la evolución. 
Bio-grafia Escritos sobre la Biologia y su Enseñanza.

Edición Extra-Ordinaria. ISSN 2027-1034 P.p 705 - 714

Memorias del VII Encuentro Nacional de Experiencias en la Enseñanza de la Biología y la Educación Ambiental y II Congreso Nacional de Investigación en la Enseñanza de la Biología

Valbuena et. al. (2010), agrupan los trabajos de de esta sub-categoría en tres tendencias:

- Instrumentalista-activista: Estrategias y ayudas para la enseñanza de conceptos particulares.

- Escenarios de educación no formal para la enseñanza de la Biología.

- Modelos pedagógicos y didácticos en la enseñanza de la Biología.

En el caso de los artículos revisados estos se agruparían en la primera tendencia, ya que usan experimentaciones, simulaciones, actividades, entre otras, para la enseñanza de conceptos específicos de la Biología. Al respecto, se puede observar que en un porcentaje menor estas experiencias llevan a la construcción de investigaciones que discuten la efectividad de la experiencia y problematizan su uso en la Enseñanza de la Biología. El hecho de que se encuentren trabajos que pasen del hecho descriptivo es un aspecto favorable, aunque como se dijo anteriormente es una pequeña muestra con respecto al total de los artículos que atienden a esta categoría.

La sub-categoría de Ideas previas/concepciones/percepciones, presenta en sus publicaciones dos tendencias; la primera hacia la indagación de dichas ideas o percepciones y la otra por la generación de actividades que permita la corrección de las mismas para la mejora en la enseñanza de un concepto especifico, es decir, que se identifica en estas ideas un obstáculo para el aprendizaje. Para ejemplificar dichas tendencias se puede observar el trabajo de Moore, R. (2007) cuyo objetivo es indagar las percepciones de estudiantes y profesores respecto a la enseñanza de la evolución. Es importante que se haya encontrado esta categoría en tanto, es una línea de investigación consolidada en la Didáctica de las Ciencias, las ideas previas/percepciones o concepciones, son para Porta (2007), teorías implícitas que tienen un cierto orden, una cierta organización, las mismas aparecen formando redes conceptuales que les son efectivas para quienes las sostienen, y son de sentido común porque les permiten dominar situaciones desde lo cotidiano.

La sub-categoría de factores personales y contextuales en la Enseñanza de la Biología retomada de Duran (2010), presenta particularmente trabajos asociados al fortalecimiento de los procesos de enseñanza en personas con problemas en el aprendizaje, haciendo alusión específicamente al trabajo realizado por Bodzin, A, et.al. (2007); que presenta el diseño de una actividad basada en un prototipo de 
Bio-grafia Escritos sobre la Biologia y su Enseñanza.

Edición Extra-Ordinaria. ISSN 2027-1034 P.p 705 - 714

Memorias del VII Encuentro Nacional de Experiencias en la Enseñanza de la Biología y la Educación Ambiental y II Congreso Nacional de Investigación en la Enseñanza de la Biología

programa web de Biología integrada, a estudiantes noveno grado que incluían dos estudiantes en programas de educación individual.

La sub-categoría Tecnologías de la información y la comunicación (TIC)presenta dos tendencias: una propone actividades que emplean las tecnologías de la información y comunicación en la Enseñanza de la Biología, tanto de manara general como en conceptos específicos; la segunda, hace referencia a la problematización de los usos de los sitios web en la enseñanza, como es el caso del trabajo elaborado por Littlefield, A. (2007) quien presenta una discusión respecto a los criterios de selección de los sitios web que se emplean en el aula .

El uso de las TIC en la Enseñanza de las Ciencias es un tema que en los últimos años ha cobrado gran importancia debido al uso casi generalizado de estas herramientas, su uso en la tiene implicaciones de distintos ámbitos: didácticas (favorecen un aprendizaje más significativo, facilitan la conexión con la vida cotidiana, contextualizan la ciencia con las relaciones CTS, interesan a los estudiantes, etc.), epistemológicas (mejoran la comprensión de la naturaleza de la ciencia y la tecno-ciencia contemporánea) y por supuesto, sociales (capacita a los ciudadanos para su participación democrática en la sociedad civil a la hora de tomar decisiones con fundamento sobre cuestiones tecno/científicas de interés social) (Maizteguiet al., 2002).

La sub-categoría de Evaluación presenta el diseño de rubricas evaluativas de conceptos Biológicos, específicamente la evolución biológica como la elaborada por Jensen, M, et.al (2007), se puede observar que la tendencia se encuentra centrada en el diseño y aplicación de las rubricas, esto hace pensar que hay una preocupación de generar nuevos instrumentos que permitan evaluar específicamente las particularidades de los conceptos, como de las habilidades propias de la actividad científica. El diseño de instrumentos evaluativos deberá responder entonces, a la visión de evaluación de los aprendizajes que se tenga, esto queda evidenciado en el momento de la aplicación del instrumento, bien sea durante el proceso o solo al finalizar las acciones de enseñanza.

Las sub-categorías de Actitudeshacia las temáticas biológicas, formación del profesorado, lenguaje y aprendizaje; reportan los porcentajes menores respecto a las sub-categorías anteriormente mencionadas.

Respecto a las sub-categorías que son estudiadas en un porcentaje notoriamente menor, es de destacar que se hace necesario aumentar los estudios que retomen estas problemáticas ya que hacen parte de los ejes centrales a la hora de emprender acciones que favorezcan los procesos de enseñanza/aprendizaje, 
Bio-grafia Escritos sobre la Biologia y su Enseñanza.

Edición Extra-Ordinaria. ISSN 2027-1034 P.p 705 - 714

Memorias del VII Encuentro Nacional de Experiencias en la Enseñanza de la Biología y la Educación Ambiental y II Congreso Nacional de Investigación en la Enseñanza de la Biología

sobre todo en los que respecta a las actitudes de los estudiantes y a la formación de maestros en Biología. Las actitudes son un componente fundamental de la personalidad de un individuo, la sola presencia del afecto es suficiente para desencadenar una tendencia a la acción (García, et.al; 2008). En consonancia con esta idea algunas de las acciones y de los problemas relacionados al aprendizaje subyacen en la actitud de los estudiantes hacia las ciencias, la Biología y las temáticas particulares.

El estudio de las actitudes es sumamente importante gracias a su capacidad de indicador y guía de la conducta, se ha sostenido que el cambio actitudinal podría ser un elemento clave para favorecer o facilitar también el cambio conceptual (Vázquez y Manassero, 1997).

Es notoria la poca frecuencia de los trabajos relacionados con la formación de maestro, solo 1 de 47 artículos presenta esta temática como su problema objeto de estudio. Las investigaciones relacionadas con la formación de profesores, son importantes, tanto para la identificación de las concepciones de los docentes respecto a la ciencia, a su conocimiento profesional, entre otros, como para el reconocimiento de su acción en aula y sus implicaciones, paralelamente dichos estudios construyen el sustento de los programas de formación inicial de profesores al detectar estrategias y falencias en los currículos que les atañe; es de resaltar que de los pocos trabajos de tipo investigativo encontrados en el presente estudio los trabajos que se ubican en esta categoría corresponden a esta nominación.

Esta temática ha sido poco frecuente no solo para el año 2007 en esta revista específicamente, Valbuena et.al (2010) sostiene que en su estudio este es de hecho una de las tendencias minoritarias de investigación. Contrario a los hallazgos se esperaría que fuese una línea de trabajo consolidada, en tanto, constituye fuertes pilares en la consolidación de los campo de conocimiento correspondes a las didácticas específicas, ya que atendería a las particulares del profesor de Biología.

\section{Conclusiones}

En conclusión, se puede ver que Respecto a la categoría tratada problemas abordados en la enseñanza de la Biología se observa una marcada tendencia hacia el estudio de los trabajos prácticos lo que se corresponde con los hallazgos realizados por el grupo CPPC en las dos fases de investigación y por los de Corrrea (2011), lo que la lleva a postularse como una línea de investigación fuerte en lo que respecta a la Enseñanza de la Biología en donde si se relaciona con 
Bio-grafia Escritos sobre la Biologia y su Enseñanza.

Edición Extra-Ordinaria. ISSN 2027-1034 P.p 705 - 714

Memorias del VII Encuentro Nacional de Experiencias en la Enseñanza de la Biología y la Educación Ambiental y II Congreso Nacional de Investigación en la Enseñanza de la Biología

otras categorías como autores podría observarse además si se esta consolidando un grupo de profesionales que le estén apuntando al estudio especifico de este tipo de trabajos.

El estudio de las categorías descritas en el apartado anterior, revela que hay aspectos de la Enseñanza de la Biología que no se han consolidado plenamente, por lo que su configuración como un campo de conocimiento está aun en emergencia, pese a esto se encontraron aspectos favorables para su establecimiento y legitimación. Por otro lado, un estudio comparativo de los estados del arte llevados a cabo al interior del grupo Conocimiento Profesional del Profesor de Ciencias, o que abarque un espectro temporal más amplio puede aportan más elementos acerca de la Didáctica de la Biología, y por ende de la emergencia del mismo.

La construcción de los Estados del Arte permiten la escogencia del material documental a estudiar y de los años que se desea hacer la investigación, lo anterior hace que los estados del arte representen un espacio potencial de investigación, en tanto el espacio queda abierto a que nuevas investigaciones alimenten los hallazgos de los estado del arte elaborados al interior del grupo de investigación Conocimiento Profesional del Profesor de Ciencias, con la escogencia de nuevas publicaciones, categorías de análisis y diferentes periodos de tiempo que ayuden a la elucidación y consolidación de unas características propias a la Enseñanza de la Biología.

Una comunidad dedicada al estudio de la Enseñanza de la Biologia es perceptible también al conocer que la revista The American BiologyTeacheres originada por una asociación de profesores de Biología. Igualmente a partir de los hallazgos de la investigación se podría decir que una parte muy importante está compuesta por docentes universitarios, investigadores y docentes universitarios. Los elementos descritos anteriormente son favorables a la consolidación de un campo de conocimiento centrado en la Enseñanza de la Biología.

\section{Referencia Bibliográficas}

ADURIZ, A; IZQUIERDO, M. (2002) Acerca de la didáctica de las ciencias como disciplina autónoma. En: Enseñanza De Las Ciencias. VOL. 1 No 3

BERNAL, S.(2009)Contribución al estado del arte del campo del conocimiento de la Enseñanza de la Biología: Journal of BiologicalEducation 2007-2008. Tesis (Especialización en Enseñanza de la Biología)- Universidad Pedagógica Nacional de Bogotá D.C., Bogotá DC 
Bio-grafia Escritos sobre la Biologia y su Enseñanza.

Edición Extra-Ordinaria. ISSN 2027-1034 P.p 705 - 714

Memorias del VII Encuentro Nacional de Experiencias en la Enseñanza de la Biología y la Educación Ambiental y II Congreso Nacional de Investigación en la Enseñanza de la Biología

BOURDIEU, P, (1996). Campo de poder campo intelectual. Editorial Montressor.

BUGALLO, A; (1995) La Didáctica de la genética, revisión bibliográfica. En: Enseñanza de las ciencias. Vol 13 № 3.Pp 379-385.

CAAMAÑO, A. (2004). Experiencias e experimentos ilustrativos, ejercicios prácticos e investigaciones. ¿Una clasificación útil para los trabajos prácticos?, En Alambique, 39, 8-19.

CORREA, M (2012). Aproximación Al Estado Del Arte Sobre Los Trabajos Prácticos En La Enseñanza De La Biología (2004-2006).Trabajo de grado para optar al título de Magister en Educación. Universidad Pedagógica Nacional. Bogotá D C.

DURAN, O. (2010) Contribución al estado del arte sobre la Enseñanza de la Biología. El caso de The American BiologyTeacher. Trabajo de grado para optar al título de Especialista en Enseñanza de la Biología. Universidad Pedagógica Nacional. Bogotá D C.

HERNÁNDEZ et.al (2011) Estado del arte de las características del campo de conocimiento de la Enseñanza de la Biología 2004-2006. Informe de proyecto de investigación,: CIUP Universidad Pedagógica Nacional.

VALBUENA, E.; AMÓRTEGUI, E.; CORREA M. y BERNAL, S (2010). Estado del arte sobre el campo de conocimiento de la Enseñanza de la Biología. Informe (Proyecto de Investigación)- Universidad Pedagógica Nacional de Bogotá DC., Bogotá DC. 